\title{
RECIDIVA LOCAL DESPUÉS DE LA PROSTATECTOMÍA RADICAL Y RADIOTERAPIA DE RESCATE.
}

\author{
Antonio 0 jea Calvo, Alberto Pérez Rodríguez, Fernando Domínguez Freire, Arturo Alonso \\ Rodrigo, Benito Rodríguez Iglesias, Juan Benavente Delgado, José M anuel Barros Rodríguez, \\ Ana González Piñeiro, Milagros 0 tero García y Víctor M uñoz Garzón.
}

Servicio de Urología. Complejo Hospitalario Universitario de Vigo. Vigo. Pontevedra. España

\begin{abstract}
Resumen.- O BJETIVO: Evaluar la eficacia de la radioterapia en el lecho prostático en pacientes con cáncer de próstata y fracaso bioquímico después de la prostatectomía radical.

M ÉTO DO S: Analizamos los resultados de 292 pacientes a los que se le practicó prostatectomía radical por cancer de próstata localizado T1-T2, entre Enero de 1992 y Junio de 2003, con un seguimiento medio de 36 meses(rango 6 meses a 12 años). Se detecta fracaso bioquímico(PSA $>0.20 \mathrm{ng} / \mathrm{ml}$ ) en $75(26 \%)$ pacientes. De los 75 pacientes con fracaso bioquímico, $9(12 \%)$ se diagnosticó de recidiva local siguiendo los siguientes criterios: a/ Primer PSA obtenido a las 6 semanas de la intervención <0.20 ng/ ml. b/ Tiempo hasta el fracaso bioquímico $>6$ meses. c/ Tiempo de
\end{abstract}

Antonio 0 jea Calvo

Hospital Universitario Xeral- C íes de Vigo c/ Pizarro, 22

36204 Vigo.

Pontevedra. (España)

e-mail: dr.ojea @telefonica.net

Trabajo recibido: 28 de junio 2004 duplicación del PSA > 6 meses. d/ Velocidad de PSA después de la prostatectomía radical < $0.75 / \mathrm{ng} / \mathrm{ml} /$ año. el $\mathrm{N}$ ivel de PSA después de la prostatectomía radical $<2.5 \mathrm{ng} / \mathrm{ml}$. Los 9 pacientes diagnosticados de recidiva local reciben una dosis media de $56.42 \mathrm{G}$ y en el lecho prostático.

RESULTADOS: De los 9 pacientes diagnosticados de recidiva local, en $8(88.8 \%)$ se obtuvo una respuesta completa durante una mediana de seguimiento de 30 meses(12-36 meses). El tiempo entre la radioterapia y la respuesta, en los pacientes con respuesta completa, fue inferior a los 3 meses en 7 pacientes y a 12 meses en 4 pacientes. No se observaron efectos adversos importantes secundarios a la radioterapia.

CONCLUSIONES: La radioterapia de rescate puede ser beneficiosa en un seleccionado grupo de pacientes con recidiva local. La cinética del PSA después de la prostatectomía radical es útil para distinguir las recidivas locales de las metástasis a distancia.

Palabras clave: Prosta tectomía radical. Recidiva local. Radioterapia de rescate.

Resumen.- O BJETIVE: To evaluate the efficacy of radiotherapy to the prostatic bed in patients with biochemical recurrence prostate cancer after radical prostatectomy.

METHODS: We analyse the outcomes of 292 patients who underwent radical prostatectomy for localized prosta te cancer T1-T2 between January 1992 and June 2003, with an average folow-up of 36 months (range 
6 months to 12 years). W e detected biochemical recurrence (PSA > $0.20 \mathrm{ng} / \mathrm{ml}$ ) in $75(26 \%)$ patients. 75 patients with biochemical recurrence, 9 (12\%) were diagnosed of local recurrence by the following criteria: a) First PSA obtained 6 weeks after radical

prostatectomy $<0.20 \mathrm{ng} / \mathrm{ml}$. b) Time to biochemical recurrence $>6$ months. c) Prostate specific antigen doubling time $>6$ months. d) Prostate specific antigen velocity after radical prostatectomy $<0.75 \mathrm{ng} / \mathrm{ml} /$ year. e) Prostate specific antigen level after radical prostatectomy $<2.5 \mathrm{ng} / \mathrm{ml}$. The 9 patients diagnosed of local recurrence received an average dose of $56.42 \mathrm{G} y$ to the prostate bed.

RESULTS: of all 9 patients with local recurrence, $8(88.8 \%)$ have complete response with a mean followup of 30 months (12-36 months). The time between the radiotherapy and the response, in patients with complete response, was lower than 3 months in 7 patients and 12 months in 1 patient. Significant adverse effects associated to radiotherapy were not observed.

CON CLUSIO N S: Salvage radiotherapy may be beneficial in selected patients with local recurrence. The characteristics of prostate specific antigen elevation are useful in distinguishing men with local recurrence from those with distant metastases.

Keywords: Radical prosta tectomy. Local recurrence. Salvage radiotherapy.

\section{INRODUCCIÓN}

La recidiva del cáncer de próstata después de la prostatectomía radical se sitúa en torno al 30\% de los pacientes. Aunque se han descrito casos a los $10 \mathrm{y}$ 15 años de la intervención, suele manifestarse entre los 19 y 24 meses de la intervención quirúrgica. Se diagnostica por la elevación del PSA por encima de $0.2 \mathrm{ng} / \mathrm{ml}$, que es lo que se denomina con el termino de fracaso bioquímico o fracaso del PSA. El análisis de la cinética del PSA o comportamiento del PSA postoperatorio es el mejor método para determinar la localización de la recidiva, clasificándola en local o metastásica. (1-7). Un tiempo hasta el fracaso del PSA superior a 6 meses predice recidiva local y menor de 6 meses predice diseminación metastásica. Una velocidad del PSA elevándose después de la cirugía a un ritmo de menos de $0.75 \mathrm{ng} / \mathrm{ml} /$ año es muy significativa de recidiva local mientras que si se eleva a una velocidad de más de $0.75 \mathrm{ng} / \mathrm{ml} /$ año es sinónimo de diseminación metastásica. Un tiempo de duplicación del PSA superior a 6 meses indica recidiva local y el inferior a 6 meses recidiva sistémica. La cantidad de PSA también es útil para hacer el diagnóstico entre recidiva local y sistémica. PSA inferior a $2.5 \mathrm{ng} / \mathrm{ml}$ es significativo de recidiva local y superior a $2.5 \mathrm{ng} / \mathrm{ml}$ de recidiva sistémica (1-11). Las técnicas por imagen disponibles actualmente, gammagrafía ósea, tomografía axial computarizada, resonancia magnética, radioinmunocentellografía, tomografía por emisión de positrones, demuestran poca utilidad en el diagnóstico precoz de la recidiva y en la localización de la recidiva (4). La biopsia del lecho prostático es poco eficaz para localizar la recidiva en pacientes con PSA inferior a $1 \mathrm{ng} / \mathrm{ml}$. El tratamiento con intención curativa de la recidiva local es la radioterapia del lecho prostático (4).

El objetivo de éste estudio es valorar la capacidad de la radioterapia de rescate para erradicar la recidiva local diagnosticada por la cinética del PSA.

\section{MATERIAL Y MÉTODOS}

Se analiza la evolución de 292 pacientes con cáncer de próstata T1-T2 a los que se le practicó prostatectomía radical retropúbica entre Enero de 1992 y Junio de 2003. El tiempo de seguimiento medio es de 36 meses (6 meses-12 años). Los tumores se reclasificaron según el sistema TN M de 2002 sexta edición de la UICC (12). En todos los pacientes se establecieron controles periódicos con determinación de PSA en sangre a las 6 semanas de la intervención y posteriormente cada 6 meses de forma indefinida. En caso de PSA superior a $0.20 \mathrm{ng} / \mathrm{ml}$ en al menos dos determinaciones se diagnosticaba al paciente de fracaso bioquímico. El PSA se determinó con el ensayo KRYPTO $R^{\circledR}$ que se fundamenta en la tecnología TRACE $^{\circledR}$ (Time Resolved Amplified Cryptate Emission).

Se detectó fracaso bioquímico en 75 pacientes lo que representa un porcentaje del $26 \%$. Se observó fracaso bioquímico en el $10 \%(2 / 20)$ de los tumores pT0, en el $11 \%(8 / 74)$ de los tumores pT2a, en el $12 \%(8 / 67)$ de los tumores pT2b, en el $21 \%(7 / 34)$ de los tumores pT2C, en el $28 \%(19 / 61)$ de los tumores pT3a, en el $74 \%(14 / 19)$ de los tumores pT3b, en el $100 \%(2 / 2)$ de los tumores PT4 y en el $100 \%(15 / 15)$ de los tumores PT2-3N 1. 
Para hacer el diagnóstico de recidiva local del cancer de próstata por la cinética del PSA postoperatorio se seleccionó a los pacientes que cumplían todos los criterios siguientes:

1. Q ue el PSA hubiese descendido a cifras insignificantes a las 6 semanas de la prostatectomía (PSA postoperatorio $<0.20 \mathrm{ng} / \mathrm{ml}$ ).

2. Q ue el tiempo hasta el fracaso bioquímico (PSA > de $0.20 \mathrm{ng} / \mathrm{ml}$ ) fuese mayor de 6 meses.

3. Que el tiempo de duplicación del PSA fuese mayor de 6 meses.

4. $Q$ ue la velocidad del PSA fuese inferior a $0.75 \mathrm{ng} / \mathrm{ml} /$ año.

5. $Q$ ue el PSA no sobrepasase la cifra de 2.5 $\mathrm{ng} / \mathrm{ml}$.

El número de pacientes con recidiva local diagnosticada por la cinética del PSA fue de 9 , lo que representa el $12 \%$ de todos los pacientes con fracaso bioquímico: $1(11.1 \%)$ se correspondía con un tumor pTa, $2(22.2 \%)$ con tumor pT2b, 2(22.2\%) con tumor pT2c, 2(22.2\%) con tumor pT3a , 1(11.1\%) con tumor pT3b y $1(11.1 \%)$ con tumor pT4a. La edad media de los pacientes con recidiva local era de 58 años que se siguieron durante un tiempo medio de 50 meses (18106). La mediana de tiempo hasta la progresión biológica era de 17 meses (12-44meses). La mediana de tiempo de duplicación del PSA era de 12 meses (1248 meses). La mediana de la velocidad de PSA en la recidiva bioquímica era de $0.25 \mathrm{ng} / \mathrm{ml} /$ año $(0.05$ 0.74). La mediana de PSA previo a la radioterapia era de 1.04(0.37-2.40) (Tabla I).

Para el tratamiento de radioterapia de rescate se utilizaron fotones de $18 \mathrm{MV}$ de acelerador lineal. El volumen blanco incluía el lecho de prostatectomía, utilizando 4 campos en caja conformados con multiláminas isocéntricas. Se administró una dosis media de radioterapia de $56.42 \mathrm{~Gy}$ en 5 fracciones semanales de 2 Gy.

Se determina el porcentaje de respuestas completas hasta cifras de PSA no significativas $($ PSA $<0.20)$ y el tiempo que transcurre desde que se administra la radioterapia y se alcanza la respuesta completa. También se calcula la duración de la respuesta completa. Para el manejo de datos y el análisis de los resultados empleamos el programa informático, SPSS para W indows, de Microsoft.

TABLA I.- Características de los 9 pacientes que recibieron radioterapia de rescate después del fracaso bioquímico y resultados. El nivel de PSA previo a la radioterapia era de 1.04(0.37-2.40); el tiempo de duplicación del PSA de 12 meses(12-48); la velocidad del PSA de $0.25 \mathrm{ng} / \mathrm{ml}(0.05-0.75)$. El número de pacientes rescatados fue de $8(88.8 \%)$ con una mediana de seguimiento de 30 meses(12-36 meses).

\begin{tabular}{|c|c|c|c|c|c|c|c|c|}
\hline Paciente & $\begin{array}{c}\text { Categoría } \\
\text { pT }\end{array}$ & Edad & Gleason & $\begin{array}{c}\text { PSA previo } \\
\text { a la } \\
\text { radioterapia } \\
\text { (ng/ ml) }\end{array}$ & $\begin{array}{c}\text { Tiempo de } \\
\text { duplicación } \\
\text { PSA } \\
\text { (meses) }\end{array}$ & $\begin{array}{c}\text { PSA } \\
\text { velocidad } \\
\text { (ng/ ml/ año) }\end{array}$ & $\begin{array}{c}\text { Respuesta } \\
\text { completa a } \\
\text { la } \\
\text { radiotera pia }\end{array}$ & $\begin{array}{c}\text { Tiempo de } \\
\text { seguimiento con } \\
\text { respuesta a la } \\
\text { radioterapia } \\
\text { (meses) }\end{array}$ \\
\hline 1 & pT3a & 48 & 6 & 1.71 & 36.00 & 0.16 & Si & 18,00 \\
2 & pT4a & 62 & 9 & 0.76 & 24.00 & 0.25 & Sí & 36,00 \\
3 & pT2c & 59 & 7 & 0.37 & 48.00 & 0.12 & Sí & 18,00 \\
4 & pT2a & 62 & 6 & 1.48 & 24.00 & 0.74 & Sí & 31,00 \\
5 & pT2b & 54 & 6 & 1.05 & 12.00 & 0.20 & Sí & 36,00 \\
6 & pT2c & 59 & 6 & 0.89 & 12.00 & 0.25 & Sí & 33,00 \\
7 & pT3a & 52 & 6 & 1.04 & 12.00 & 0.27 & Sí & 30,00 \\
8 & pT2b & 63 & 8 & 0.62 & 12.00 & 0.05 & Sí & 12,00 \\
9 & pT3b & 60 & 8 & 2.40 & 12.00 & 0.74 & No & N 0-respuesta \\
\hline
\end{tabular}




\section{RESULTADOS}

El número de pacientes con respuesta completa a la radioterapia local fue de $8(88.8 \%)$. El tiempo que transcurrió desde que se administró la radioterapia hasta que se alcanzaron cifras de PSA $<$ de 0.20 $\mathrm{ng} / \mathrm{ml}$ fue inferior a 3 meses en 7 pacientes y de 12 meses en 1 paciente. Los pacientes que respondieron lo hicieron durante una mediana de tiempo de 30 meses (12 -36 meses) y no se observaron nuevas recaídas en los pacientes que respondieron. Los efectos secundarios de la radioterapia se manifestaron con sintomatología intestinal, rectal o vesical leve en $5(56 \%)$ de los pacientes que fueron controlados con tratamiento sintomático (Tabla I) (Figura 1).

\section{COMENTARIOS}

El tratamiento de la recidiva local del cáncer de próstata después de la prostatectomía radical es la radioterapia del lecho prostático con intención curativa (13). Hasta la actualidad los métodos más frecuentemente utilizados con la pretensión de localizar la recidiva tumoral han sido: el análisis del comportamiento del PSA después de la intervención o cinética del PSA postoperatorio, la gammagrafía ósea, la tomografía axial computarizada, la resonancia magnética, la radioinmunocentellografía, la tomografía por emisión de positrones y la biopsia del lecho prostático.

Cinética del PSA postoperatorio.- La determinación en sangre del PSA es el método más simple y específico para detectar la presencia de tumor residual después de la prostatectomía radical. El primer control se suele realizar a las 6 semanas de la intervención, para evitar falsos positivos. Por que hay que tener en cuenta que la vida media del PSA es de 2.6 días y que durante la intervención quirúrgica existe la posibilidad que una gran cantidad de PSA se libere y pase a la sangre. Si el tumor esta localmente avanzado o el G leason es elevado los controles se continúan semestralmente los 2 ó 3 primeros años y posteriormente cada año de forma indefinida. Si el tumor esta localizado y el Gleason es intermedio o bajo, los controles de PSA pueden practicarse anualmente de forma indefinida $(4,14,15)$. A unque no existe un consenso que defina cual es el nivel de PSA insignificante, la idea más generalizada es que una cifra de PSA de 0.10 inferior, con los ensayos convencionales, se debe a un artefacto que puede provenir de las glándulas periuretrales. $\mathrm{N}$ iveles de PSA de $0.2 \mathrm{ng} / \mathrm{ml} 0$ superiores son patológicos e indican presencia de tumor residual. $\mathrm{N}$ iveles de PSA entre 0.1 y $0.2 \mathrm{ng} / \mathrm{ml}$ son cifras que están en terreno de nadie, que solo se consideran patológicos a posteriori, cuando el PSA sobrepasa los $0.20 \mathrm{ng} / \mathrm{ml}$ en los controles posteriores. Entonces se utiliza para estimar el tiempo de duplicación de PSA (4).

El análisis de la cinética del PSA postoperatorio se consideró una buena forma de localizar el lugar de la recidiva. Partin AW et als en 1994 (9) analizaron la utilidad para localizar el lugar de la recidiva del tiempo que transcurría desde la prostatectomía hasta el fracaso del PSA y de la velocidad de ascenso del PSA una vez que se había producido el fracaso bioquímico. De una serie de 1058 prostatectomías radicales los autores siguieron a 51 pacientes con fracaso bioquímico, hasta la aparición de recidiva local o la aparición de metástasis. De los 51 pacientes, 16(31\%) recidivaron localmente y $35(69 \%)$ lo hicieron con metástasis a distancia. El intervalo de tiempo desde la

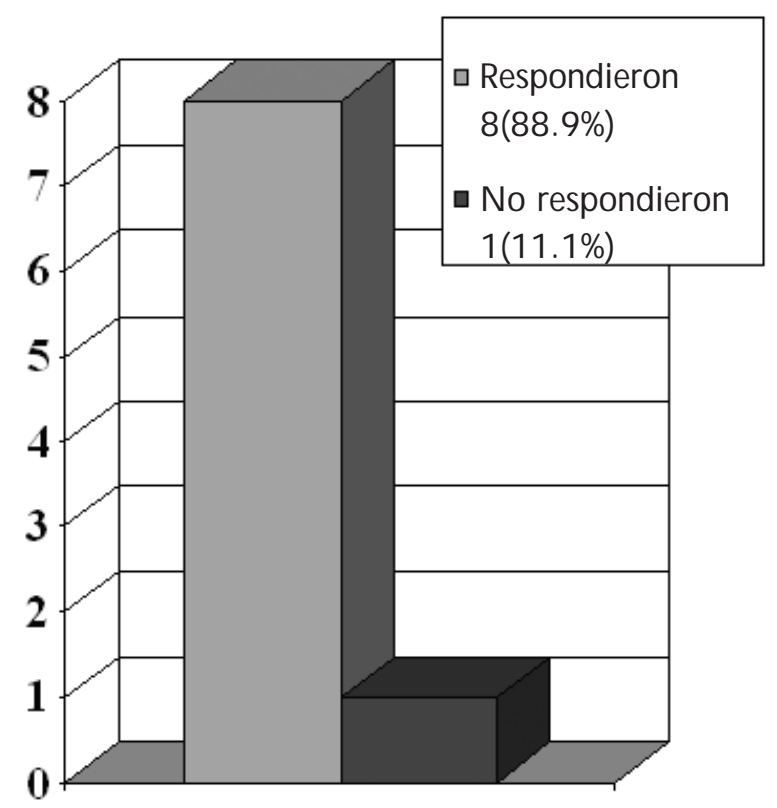

Figura 1.- $8(88.8 \%)$ de los 9 pacientes seleccionados por la dinámica del PSA postoperatorio respondieron a la radioterapia de rescate en el lecho prostático y no se detectó recaída en un tiempo de seguimiento de 30 meses(1 $2-36$ meses). 
prostatectomía radical hasta el fracaso del PSA fue más largo en los pacientes con recidiva local que en los que tenían metástasis. $N$ ingún paciente con recidiva local registró un PSA detectable dentro de los 6 meses siguientes a la cirugía frente al $50 \%$ de los que desarrollaron metástasis a distancia. En el mismo trabajo, también se observó que la velocidad de ascenso del PSA era distinta en los pacientes con recidiva local y en los pacientes con metástasis. En el $94 \%$ de los pacientes con recidiva local el PSA crecía a un ritmo inferior a $0.75 \mathrm{ng} / \mathrm{ml} /$ año frente al $46 \%$ de los pacientes que presentaban metástasis a distancia. El tiempo de duplicación del PSA fue analizado por diversos autores, con intención de localizar la recidiva tumoral. Patel A et als (10) analizaron 77 pacientes con fracaso de PSA después de la prostatectomía radical. En 33 pacientes el tiempo de duplicación del PSA era inferior a 6 meses y en 44 era superior a 6 meses. En $11(33 \%)$ de los pacientes con tiempo de duplicación de PSA inferior a 6 meses observaron metástasis a distancia frente a $1(3 \%)$ que presentaba recidiva local. Cuando el tiempo de duplicación del PSA era superior a los 6 meses solamente en $1(2 \%)$ de los pacientes se observó metástasis a distancia frente a $8(18 \%)$ que presentaban recidiva local. Pound $C R$ et als(16) observaron que un tiempo de duplicación del PSA inferior a los 10 meses era la variable que mejor predecía la aparición de enfermedad metastásica. Roberts SG et als (17) concluyen en un análisis univariante y multivariante que el tiempo de duplicación del PSA era la variable con más capacidad para discernir entre la recidiva local y la enfermedad metastásica después de la prostatectomía radical. El valor del nivel de PSA en sangre para predecir el lugar de la recidiva fue estudiado por W u JJ et als en 1995 (11).

A nalizaron los resultados de la radioterapia local en un grupo de 53 pacientes con fracaso bioquímico, 27 con niveles de PSA inferiores a $2.5 \mathrm{ng} / \mathrm{ml}$ y 26 con niveles de PSA superiores a $2.5 \mathrm{ng} / \mathrm{ml}$. Vieron que $14(52 \%)$ de los pacientes con PSA menor de 2.5 respondían a la radioterapia mientras que solo respondían $2(8 \%)$ de los pacientes con niveles de PSA superiores a $2.5 \mathrm{ng} / \mathrm{ml}$.

Técnicas por imagen.- Diferentes técnicas por imagen han sido evaluadas para detectar y localizar la recidiva después de la prostatectomía radical. La gammagrafía ósea fue estudiada por Cher ML et als en 1998 (18) en una serie de 93 pacientes. La posibilidad de que la gammagrafía ósea fuese positiva era inferior al $5 \%$ en pacientes con PSA por debajo de 40 $\mathrm{ng} / \mathrm{ml}$. La tomografía axial computarizada fue utilizada por O Ider RA et als en 1995 (19) comparando las imágenes del lecho quirúrgico de 11 pacientes con fracaso del PSA con otros pacientes sin fracaso del PSA que actuaban como control. No observaron diferencias significativas en las imágenes de los lechos quirúrgicos de los 2 grupos. Kramer $\mathrm{S}$ et als en 1997 (20) observaron que la tomografía axial computarizada solamente diagnosticaba el $36 \%$ de las recidivas locales confirmadas con biopsia. La tomografía axial computarizada sólo podía reconocer las recidivas locales de más de $2 \mathrm{~cm}$. La radioinmunocentellografía fue evaluada por Kahn D et als en 1998 (21). Comunicaron los resultados del análisis de 183 pacientes y la radioinmunocentellografía detectó recidiva en la celda prostática sólo en el $35 \%$ de los casos en los que ya estaba confirmada la recidiva local por biopsia. Utilizando la biopsia como patrón oro, la sensibilidad de la radioinmunocentellografía era del $49 \%$ y la especificidad del $77 \%$, con un valor predictivo positivo del $50 \%$ y negativo del $70 \%$. Raj GV et als en 2002 (22) concluyeron en su análisis que la radioinmunocentellografía era superior a la tomografía axial computarizada y a la gammagrafía ósea para detectar metástasis. La tomografía por emisión de positrones fue evaluada por Sanz G et als en 1999 (23). Ésos autores observaron en la tomografía por emisión de positrones más eficacia para mostrar recidiva local que la tomografía axial computarizada y menos eficacia que la gammagrafía ósea para detectar recidivas a distancia.

Biopsia del lecho prostático.- La biopsia ecodirigida del lecho prostático y de la anastomosis de la unión vesicouretral es un procedimiento diagnóstico de la recidiva local del cáncer de próstata después de la prostatectomía radical. Sin embargo, bajos volúmenes de la enfermedad que pueden ser detectados con niveles de PSA muy bajos pueden pasar desapercibidos y no ser diagnosticados con una biopsia del lecho prostático. Una biopsia positiva no excluye enfermedad sistémica ni una biopsia negativa excluye enfermedad local (4). Según los análisis de Connolly JA et als de 1996 (24) y Saleem MD et als de 1998 (25) solamente un $25 \%$ de los pacientes diagnosticados de recidiva local con niveles de PSA inferiores a $1 \mathrm{ng} / \mathrm{ml}$ presentaban biopsia positiva del lecho prostático. En ése sentido, Lange PH et als en 1990 (26) informaron de los resultados de la radioterapia local de rescate en 29 
pacientes con fracaso de PSA después de la prostatectomía radical, 18 pacientes con biopsia de la unión vesicouretral positiva y 10 pacientes con la biopsia de la unión vesicouretral negativa. En el grupo de pacientes con biopsia positiva respondieron a la radioterapia $16(89 \%)$ de los pacientes y en el grupo con biopsias negativas respondieron $7(70 \%)$ de los pacientes, sin diferencias significativas entre los dos grupos.

Los pacientes con una cinética de PSA favorable, en la linea de los parámetros que hemos seleccionado en nuestro estudio, son buenos candidatos para recibir con posibilidades de éxito radioterapia en la celda prostática (27). La dosis de radiación recomendada por la Sociedad Americana de Radioterapia y O ncología es de $64 \mathrm{G}$ y o ligeramente superior, administradas en fracciones de 1.8 a $2 \mathrm{G}$ y (28). Los resultados libres de fracaso bioquímico de la radioterapia de rescate son muy variables, dependiendo de múltiples factores, y pueden estar según las series a nalizadas por $\mathrm{N}$ elson et als en 2003 (4) entre el 0 y el $55 \%$, con seguimientos que oscilan entre 3 y 10 años. En nuestro estudio los resultados libres de fracaso bioquímico con radioterapia de rescate en pacientes muy seleccionados fueron del $88.8 \%$ con una mediana de seguimiento de 30 meses.

\section{CONCLUSIONES}

La radioterapia de rescate del lecho prostático es eficaz en casos muy seleccionados de pacientes con recidiva bioquímica después de la prostatectomía radical. El análisis de la cinética del PSA o comportamiento del PSA postoperatorio es un buen método para distinguir entre recidiva local y recidiva sistémica e indicar tratamiento con radioterapia en el lecho prostático, con intención curativa.

\section{BIBUOGRAFIA y LECTURAS RECOMENDADAS (*lectura de interés y **lectura fundamental)}

**1. POUND, C.R.; PARTIN, A.W.; EPSTEIN, J.I. y cols.: "Prostate-specific antigen after anatomic radical retropubic prostatectomy. Patterns of recurrence and cancer control." Urol. Clin. North Am., 124: 395. 1997.

*2. LOBERG, R.D.; FIELHAUER, J.R.; PIENTA, B.A. y cols.: "Prostate-specific antigen doubling time and survival in patients with advanced metastatic prostate cancer". Urology, 62: 128. 2003.
3. CHATTA, G.: " Multiplex biomarker approach for determining risk of prostate-specific antigen-defined recurrence of prostate.” J. Urol. Oncol., 21: 488. 2003.

*4. NELSON, J.B.; LEPOR, H.: "Prostate cancer: radical prostatectomy”. Urol. Clin. North Am., 30: 703. 2003.

5. WARD, J.F.; BLUTE, M.L.; SLEZAK, J. y cols.: "The long-term clinical impact of biochemical recurrence of prostate cancer 5 or more years after radical prostatectomy". J. Urol., 170: 1872. 2003.

6. SCHEEPE, J.R.; VIS, N.; MICKISCH, G.H.: "Recurrence after radical prostatectomy for organ-confined prostate cancer”. Onkologie, 26: 330. 2003.

7. MOUL, J.W.: "Variables in predicting survival based on treating "PSA-only" relapse". Urol. Oncol., 21: 292. 2003.

8. CANNON, G.M.; WALSH, P.C.; PARTIN, A.W.: "Prostate-specific antigen doubling time in the identification of patients at risk for progression after treatment and biochemical recurrence for prostate cancer". Urology, 62: 2. 2003.

**9. PARTIN, A.W.; PEARSON , J.D.; LANDIS, P.K. y cols.: "Evaluation of serum prostate-specific antigen velocity after radical prostatectomy to distinguish local recurrence from distant metastases”. Urology, 43: 649. 2003.

**10. PATEL, A.; DOREY, F.; FRANKLING, J. y cols.: "Recurrence patterns after radical retropubic prostatectomy: clinical usefulness of prostate specific antigen doubling times and log slope prostate specific antigen”. J. Urol., 158: 1441. 1997.

**11. WU, J.J.; KING, S.C.; MONTANA, G.S. y cols.: "The efficacy of postprostatectomy radiotherapy in patients with an isolated elevation of serum prostate-specific antigen”. Int. J. Radiat. Oncol. Biol. Phys., 132: 317. 1995.

12. SOBIN, L.H.; WITTEKIND, C.H.: "TNM Clasificación de los tumores malignos". Pulso Ediciones, 26. 2002.

13. PISANSKY, T.M.; KOZELSKY, T.F.; MYERS, R.P. y cols.: "Radiotherapy for isolated serum prostate specific antigen elevation after prostatectomy for prostate cancer". J. Urol., 63: 845. 2000.

14. HAAB, F.; MEULEMANS, A.; BOCCON-GIBOD, L. y cols.: "Clearance of serum PSA after open surgery for benign prostatic hypertrophy, radical cystectomy, and radical prostatectomy". Prostate, 26: 334. 1995.

15. BRAWER, M.K.; KIRBY, R.: “Antígeno prostático específico”. Ediciones Médicas SL, 7. 2001.

16. POUND, C.R.; PARTIN, A.W.; EISENBERGER, M.A. y cols.: "Natural history of progression after PSA elevation following radical prostatectomy". JAMA., 1281: 1591. 1998.

**17. ROBERTS, S.G.; BLUTE, M.L.; BERGSTRALH, E.J. y cols.: "PSA doubling time as a predictor of clinical progression after biochemical failure following radical prostatectomy for prostate cancer". Mayo Clin. Proc., 76: 576. 2001.

*18. CHER, M.L.; BIANCO, F.J.Jr.; LAM, J.S. y cols.: "Limited role of radionuclide bone scintigraphy in patients with prostate specific antigen elevations after radical prostatectomy". J. Urol., 160: 1387. 1998.

*19. OLDER, R.A.; LIPPERT, M.C.; GAY, S.B. y cols.: "Computed tomography appearance of the prostatic 
fossa following radical prostatectomy". Acad. Radiol., 2: 470. 1995.

*20. KRAMER, S.; GORICH, J.; GOTTFRIED, H.W. y cols.: "Sensitivity of computed tomography in detecting local recurrence of prostatic carcinoma following radical prostatectomy”. Br. J. Radiol., 70: 995. 1997.

21. KAHN, D.; WILLIAMS, R.D.; MANYAK, M.J. y cols.: "111Indium-capromab pendetide in the evaluation of patients with residual or recurrent prostate cancer after radical prostatectomy". The ProstaScint Study Group. J. Urol., 159: 2041. 1998.

22. RAJ, G.V.; PARTIN, A.W.; POLASCIK, T.J.: "Clinical utility of indium 111-capromab pendetide immunoscintigraphy in the detection of early, recurrent prostate carcinoma after radical prostatectomy". Cancer, 94: 987. 2002.

23. SANZ, G.; ROBLES, J.E.; JIMÉNEZ,M. y cols.: "Positron emission tomography with 18fluorine-labelled deoxyglucose: utility in localized and advanced prostate cancer". BJU Int., 84: 1028. 1999.

*24. CONNOLLY, J.A.; SHINOHARA, K.; PRESTI, J.C. Jr. y cols.: "Local recurrence after radical prostatectomy: characteristics in size, location, and relationship to prostate-specific antigen and surgical margins". Urology, 47: 225. 1996.

*25. SALEEM, M.D.; SANDERS, H.; ABU EL NASER, M. y cols.: "Factors predicting cancer detection in biopsy of the prostatic fossa after radical prostatectomy". Urology, 151: 283. 1998.

*26. LANGE, P.H.; LIGHTNER, D.J.; MEDINI, E. y cols.: "The effect of radiation therapy after radical prostatectomy in patients with elevated prostate specific antigen levels". J. Urol., 144: 927. 1990.

27. NUDELL, D.M.; GROSSFELD, G.D.; WEINBERG, V.K. y cols.: "Radiotherapy after radical prostatectomy: treatment outcomes and failure patterns". Urology, 54: 1049. 1999.

28. COX, J.D.; GALLAGHER, M.J.; HAMMOND, E.H. y cols.: "Consensus statements on radiation therapy of prostate cancer: guidelines for prostate re-biopsy after radiation and for radiation therapy with rising prostate-specific antigen levels after radical prostatectomy". American Society for Therapeutic Radiology and Oncology Consensus Panel. J. Clin. Oncol., 17: 1155. 1999. 